\title{
FACTORES LABORALES Y ESTRÉS PERCIBIDO EN LOS CONDUCTORES DE BUSES MEDELLÍN, 2017
}

\begin{abstract}
Catalina González Penagos ${ }^{1}$, Diana Verónica Giraldo López², Melissa Cano
\end{abstract} Gómez ${ }^{3}$, Diana Isabel Ramírez Pérez ${ }^{4}$

\section{Resumen}

Introducción. el estrés es una respuesta adaptativa física y psicológica ante las demandas y amenazas del entorno. Sumar el estrés al espacio laboral, tiene consecuencias sobre la calidad de vida laboral de los trabajadores. Métodos. estudio observacional descriptivo de corte transversal. Muestra de 278 conductores, con un nivel de confianza del $98 \%$, un margen error del 5\% y una proporción esperada del $50 \%$. Para la selección de los participantes se realizó un muestreo aleatorio simple. Se utilizó la escala de la EEP-10. Se realizó un análisis univariado y bivariado. Resultados. se encontró una correlación positiva y significativa $(0,232$ y valor $\mathrm{p}=0,000)$ entre la edad y el porcentaje de no control de estrés y asociación entre la variable porcentaje de control de estrés con la ruta de buses que conduce el conductor (valor $\mathrm{p}=0,019)$. Conclusiones. los conductores de buses están expuestos a una serie de condiciones desfavorables que pueden poner en peligro su salud y generar algún tipo de estrés.

Mg. Salud Pública, Fundación Universitaria Autónoma de las Américas. catalina. gonzalez@uam.edu.co

²Odontóloga, Fundación Universitaria Autónoma de las Américas.diana.giraldo@ uam.edu.co

${ }^{3}$ Odontóloga, Fundación Universitaria Autónoma de las Américas. melissacanogomez@gmail.com

${ }^{4}$ Estudiante de Odontología, Fundación Universitaria Autónoma de las Américas. 


\section{LABOR FACTORS AND STRESS PERCEIVED IN BUS DRIVERS IN MEDELLÍN, 2017}

Catalina González Penagos, Diana Verónica Giraldo López, Melissa Cano Gómez , Diana Isabel Ramírez Pérez

\section{Abstract}

Introduction. Stress is an adaptive physical and psychological response to the demands and threats of the environment. Add stress to a workplace, has consequences on the quality of work environment for workers. Methods. Descriptive, cross-sectional observational study. Sample of 278 subjects, with a confidence level of $98 \%$, an error margin of $5 \%$ and an expected proportion of $50 \%$. In order to select the participants, a simple random sampling was carried out. The scale of the EEP-10 was applied. A univariate and bivariate analysis was performed. Results. A positive and significant correlation $(0.232$ and $\mathrm{p}$ value $=0.000)$ was found between age and the percentage of nonstress control, and association between the percentage of stress control variable and the bus route taken by drivers ( $\mathrm{p}$ value). $=0.019$ ). Conclusions. Bus drivers are exposed to different unfavorable conditions that can endanger their health and generate some kind of stress. 


\section{FATORES DE TRABALHO E ESTRESSE PERCEBIDOS NOS CONDUCTORES DE ÔNIBUS DE MEDELLIIN, 2017}

Catalina González Penagos, Diana Verónica Giraldo López, Melissa Cano Gómez, Diana Isabel Ramírez Pérez

\section{Resumo}

Introdução. $\mathrm{O}$ estresse é uma resposta física e psicológica adaptativa às demandas e ameaças do meio ambiente. Adicionar estresse ao local de trabalho, tem consequências sobre a qualidade de vida no trabalho dos empregados. Métodos. Estudo observacional descritivo, transversal. Amostra de 278 motoristas, com nível de confiança de $98 \%$, margem de erro de $5 \%$ e proporção esperada de $50 \%$. Para a seleção dos participantes, foi realizada uma amostragem aleatória simples. A escala do EEP-10 foi utilizada. Uma análise univariada e bivariada foi realizada. Resultados. Uma correlação positiva e significativa $(0,232$ e valor-p $=0,000$ ) entre a idade e a percentagem de no controle do estresse e associação entre a variável porcentagem de controle de estresse com a rota de ônibus assignada ao motorista (valor-p $=0,019$ ). Conclusões. Os motoristas de ônibus são expostos a uma série de condições desfavoráveis que podem comprometer sua saúde e gerar algum tipo de estresse. 


\section{Introducción}

El interés por el estrés, sus características y consecuencias ha incrementado en años recientes. Por definición, el estrés hace referencia a un proceso complejo, multivariado y representativo de un sistema de retroalimentación; el estrés es, entonces, el resultado de una relación entre el individuo y el entorno que es evaluado como amenazante o desbordante de los recursos que posee y que pone en peligro su bienestar. De la misma forma, se sobrentenderá que el término estrés empleado en adelante hace referencia al estrés disfuncional o distrés, que es aquel que produce una sobrecarga de trabajo no asimilable que desencadena un desequilibrio fisiológico y psicológico, ocasionando reducción en la productividad del individuo, aparición de enfermedades psicosomáticas y envejecimiento acelerado $(1,2)$

Es difícil considerar alguna ocupación, profesión $u$ oficio que no implique estrés, dada la rapidez y las exigencias en las cuales están inmersas las personas. El estrés laboral es reconocido actualmente tanto como una amenaza para la salud de los trabajadores de diversos oficios como para el buen funcionamiento de las organizaciones en la que trabajan(3). Sumar el estrés, al espacio laboral, tiene consecuencias sobre la calidad de vida laboral de los trabajadores y sobre la productividad de las organizaciones. Por esta razón, es en la actualidad un tema de interés central para las sociedades y para los gobiernos $(3,4,5)$.

El estrés laboral resulta de la interacción entre el trabajador y las condiciones de trabajo. Las condiciones de trabajo estresantes están asociadas a riesgos psicosociales, tales como trabajos muy demandantes o muy poco demandantes, trabajos con un ritmo rápido o bajo presión, falta de control sobre las cargas y procesos de trabajo, carencia de apoyo social o de colegas y/o supervisores de trabajo, discriminación, aislamiento, acoso psicológico, falta de participación en la toma de decisiones, comunicación o flujo de información pobres, inseguridad laboral, ausencia de oportunidades de crecimiento, de avance o de promoción, horarios irregulares (especialmente turnos de trabajo) y exposición a condiciones físicas desagradables o peligrosas sin control alguno sobre dichas condiciones(6).

El estrés en el trabajo genera en las empresas una serie de consecuencias negativas en su productividad, al igual que en la motivación, satisfacción y compromiso laboral de sus colaboradores. Se asocia con un incremento de accidentes, ausentismo, incapacidades y enfermedades ocupacionales. Igualmente, propicia más conflictos interpersonales en el trabajo y en la familia y un mayor índice de errores; incide en la toma de decisiones y en el bajo rendimiento ocupacional y causa un distanciamiento afectivo con clientes, un aumento en los gastos en materia de salud y rotación de personal, entre otros aspectos(7).

Los conductores de transporte público urbano (TPU) tienen riesgos de salud superiores a los experimentados por otras ocupaciones. Debido a la exposición frecuente, el estrés ocupacional es considerado como el factor de riesgo 
psicolaboral de mayor impacto en los conductores de TPU. Este estrés es causado por el alto tráfico vehicular y los conflictos en el tránsito urbano, el cumplimiento de las metas económicas diarias asociadas al volumen de pasajeros transportados, la presión por cumplir en el tiempo estipulado, los trayectos de las diferentes rutas asignadas y la gran variedad de actividades que ejecutan durante sus recorridos. Junto con el estrés, el sobrepeso y la obesidad tienen mayor prevalencia en conductores con dos o más años de labor en la prestación de sus servicios $(8,9,10)$. Por otro lado, los conductores de transporte público ejecutan varias tareas de manera simultánea; en promedio, son más de 200 tareas por hora; entre ellas: parar, arrancar, mirar señales, abrir y cerrar puertas, frenar, cobrar, controlar interruptores, mirar por los espejos, conversar y girar el volante(11,12). Además, los conductores están expuestos a ruido, vibración, alta densidad del tráfico y a continuas paradas del automotor.

Esta situación contribuye a que el trabajador se sienta "estresado" física y psicológicamente, e incluso "quemado por el trabajo" (burnout). La población ocupada presenta, pues, una alta carga mental debido a un ritmo de trabajo alto, con importantes niveles de presión sobre el tiempo de trabajo. La situación se agrava con la percepción de una creciente carencia de autonomía, esto es, un escaso margen para participar en la planificación del trabajo y en la toma de decisiones(13).

Debido a los cambios generados por la globalización y las nuevas formas de tra- bajo, los trabajadores en los países en desarrollo deben hacer frente a un aumento en el estrés laboral. A pesar de que se han realizado investigaciones en estos países, más específicamente en el caso de América Latina, existe poca conciencia sobre el estrés laboral e insuficientes recursos para combatirlo(6). Una teoría sólida del estrés psicológico debe ser capaz de ayudar a comprender las variaciones en la forma en que los individuos evalúan las transacciones adaptativas con sus entornos. El afrontamiento es un esfuerzo cognitivo y conductual que permitirá a las personas gestionar las demandas que gravan o exceden sus recursos personales (1).

\section{Objetivos}

\section{Objetivo general}

Determinar los factores laborales y el estrés percibido de los conductores de buses de una empresa de la ciudad de Medellín, 2017.

\section{Objetivos específicos}

Describir las características sociodemográficas de los conductores de buses.

Identificar los factores laborales de la población de estudio.

Establecer el estrés percibido de los conductores de buses.

Analizar la posible correlación de algunas características sociodemográficas y factores laborales con el estrés. 


\section{Materiales y métodos}

\section{Diseño}

Se realizó un estudio observacional descriptivo de corte transversal. La población estuvo conformada por 560 conductores y una muestra de 278 conductores de buses. Para la muestra se hizo un cálculo no probabilístico con un nivel de confianza del $98 \%$, un margen error del 5\% y una proporción esperada del 50\%. Se realizó un muestreo aleatorio simple para la selección de los participantes. Los datos fueron recolectados de junio a diciembre del año 2017.

Se utilizó una encuesta autodiligenciada y acompañada por el profesional, fue calibrada previamente con una prueba piloto e incluyó variables relacionadas con características sociodemográficas, factores laborales y variables relacionadas con el estrés. Para medir la percepción de estrés psicológico, es decir, la medida en que las situaciones de la vida cotidiana se aprecian como estresantes, se utilizó la escala en versión en castellano de la EEP-10 usada por Remor(11) y propuesta por Cohen et al (14).

El test está formulado en escala Likert con 10 preguntas, que tienen cinco opciones de respuesta $(0=$ nunca, $1=$ casi nunca, 2 = de vez en cuando, 3 = a menudo y $4=$ muy a menudo). Está organizada en dos factores, el primer factor congrega los ítems $1,2,3,6,9$ y 10 , que se consideran negativos y muestra la percepción de pérdida de control y sus consecuencias. El segundo factor congrega los ítems 4, 5, 7 y 8, que se consideran positivos y representan la autoeficacia o control de las situaciones estresantes. La sumatoria produce la puntuación total de la escala con rango entre 0 y 40 puntos. Entre mayor puntuación, mayor presencia de estrés y no se ha definido la presencia de punto de corte $(15,16)$.

El procesamiento de los datos fue realizado en el paquete estadístico PRISM ${ }^{\odot}$ (versión 6.07, junio 12 de 2015, con licencia de la Fundación Universitaria Autónoma de las Américas). Se realizó un análisis univariado y bivariado; en el univariado se hallaron las medidas de frecuencia absoluta y relativa, al igual que medidas descriptivas de resumen como promedio, mediana, moda y desviación estándar (DE). En el análisis bivariado se relacionaron las variables sociodemográficas con los factores laborales y la escala de estrés percibido, según la prueba estadística Chi-cuadrado asociada con nivel de significación menor del 5\% y correlación de Pearson.

El presente estudio fue aprobado por el Comité de Ética en Investigación institucional (CEI) (Acta 29 del 31 de enero de 2017), y se solicitó consentimiento informado escrito a los conductores, previa realización de la encuesta, con base en la Resolución 8430 de 1993 del Ministerio de Salud(17) que reglamenta la investigación en seres humanos. Además, según la misma norma, esta investigación se clasificó de riesgo mínimo.

\section{Resultados}

Participaron en el estudio 278 conductores de buses, con una edad promedio de 36,53 años ( $\mathrm{DE}=11,86$ años). La edad mínima fue 18 años y la máxima fue 68 . 
De los conductores, el 99,6\% (277) eran hombres y el $0,36 \%$ (1) era mujer.

Por estado civil, predominaron los que convivían en unión libre, un 38,1\% (106), seguidos de los casados, con un $29,1 \%$ (81) y los solteros, con un $28,4 \%$ (79). Respecto al nivel de escolaridad, se observó que el aspecto predominante eran los conductores que terminaron el bachillerato, un 45,3\% (126), mientras que el $18,7 \%$ (52) tenían primaria completa e incompleta; solamente el 6,84\% (19) tenían un nivel educativo que oscilaba entre técnico, tecnólogo y universitario incompleto.

Del total de conductores participantes, el 79,1\% (220) tenían hijos. Frente a la tipología familiar, el 60,3\% (166) hacían parte de una familia nuclear.

\section{Factores laborales}

Se abordaron características de los factores laborales de los conductores, se halló que el 80\% (225) de los conductores son fijos y han estado en promedio en la empresa 9,5 años. Se les preguntó a los conductores sobre las horas laboradas en un día y el 88,09\% (244) respondió que trabajan más de 12 horas. Frente al tiempo que tienen de descanso, el 41,7\% (116) descansa una vez a la semana, y el $56,3 \%$ (156) considera que el tiempo de descanso es suficiente, mientras que el $43,6 \%$ (121) plantea que no es suficiente.

Se indagó sobre la actitud que tenía el conductor al realizar su labor y como esta se veía afectada por varias situaciones y se encontró que más de la mitad se sentía afectado por el chequeo de tarjeta, pernota y el calor. Frente a como considera su relación con el jefe inmediato, el 56,1\% (156) expresó que es excelente (Tabla 1).

Respecto a la percepción de las relaciones con sus compañeros de trabajo, el $52,8 \%$ (147) respondió que sus relaciones eran buenas. El 59,3\% (165) de los conductores considera que son buenas las relaciones con los pasajeros. Frente a si les gustaría tener otra posibilidad de empleo, el 71,8\% (199) respondió que sí.

Tabla 1. Distribución porcentual de las respuestas de los factores laborales de los conductores.

\begin{tabular}{l|c|c}
\hline \multicolumn{1}{c|}{ Variable } & $\begin{array}{c}\text { Frecuencia } \\
\text { absoluta }\end{array}$ & Porcentaje (\%) \\
\hline $\begin{array}{l}\text { Tipo de conductor } \\
\text { Conductor fijo }\end{array}$ & 225 & 80,9 \\
Conductor auxiliar & 52 & 18,7 \\
& & \\
Actitud se ve afectada & & 25 \\
Chequeo tarjeta y pernota & 71 & 22 \\
Calor & 62 & 20 \\
Congestión vehicular & 56 & 14 \\
Guerra del centavo & 39 &
\end{tabular}


Relación con el jefe inmediato

Excelente

\section{Conduce con calma y paciencia}

\section{Estrés percibido}

Se pretendió conocer si al encuestado alguna vez se le había diagnosticado algún problema de salud mental. Se encontró que al 8,7\% (24) de los conductores sí se les había diagnosticado; el 59,2\% (16) expresa que fue diagnosticado hace años, el 37\% (10) fue diagnosticado hace meses y solo uno 3,7\% (1) dijo haber recibido diagnóstico entre uno y treinta días anteriores.

El 30,6\% (84) de los encuestados manifestó haber sido víctima de algún evento violento durante su trabajo. Del total de los conductores que han sido víctimas de algún evento violento, el 56,4\% (66) ha sido de robo; el 15,3\% (186) por amenaza del tráfico vehicular y el 12,8\% (15) ha sido víctima de dos eventos a la vez, como amenaza por chantaje, robo y amenaza del tráfico vehicular.

Con respecto a la escala de estrés percibido, se halló que el promedio de la calificación fue del $69 \%$ de respuestas que indican que no hay control de estrés. Por lo menos el 50\% de los conductores tienen una calificación de no control del estrés, en la escala de estrés percibido, del 70\% o más.

En cada uno de los ítems de la escala de estrés percibido, se encontró que el 30,2\% (84) algunas veces ha estado afectado por algo que ha ocurrido inesperadamente, mientras el 2,8\% (8) expresó que a menudo y muy a menudo ha estado afectado. Con respecto a si se ha sentido incapaz de controlar las cosas importantes en su vida, el 20\% (56) expresó que algunas veces sí se había sentido incapaz.

Se encontró que el 42,8\% (119) de los conductores, señaló que algunas veces se ha sentido nervioso o estresado. Respecto a su capacidad para manejar sus problemas, se halló que el 35,6\% (99) nunca ha estado seguro y el $37,41 \%$ (104) casi nunca ha estado seguro (Tabla 2).

Se quiso conocer sobre la frecuencia para conocer si ellos sentían que las cosas les salían bien y se evidenció que el 
$40,7 \%$ (113) consideran que casi nunca les va bien, seguido del 34,6\% (96) que expresa que nunca les va bien.

Con relación a si los conductores han sentido que no podían afrontar todas las cosas que tenía que hacer, el 37,7\% (105) a menudo ha podido afrontar las cosas que tenía que hacer, seguido del $34,1 \%$ (95) que declara que muy a menudo. El 40,4\% (112) dice que casi nunca ha podido controlar las dificultades de su vida, mientras el 38,2\% (106) nunca las ha podido controlar (Tabla 2).

Respecto a la frecuencia que han sentido los conductores de tener todo bajo control, se evidenció que el 42,9\% (119) sienten que casi nunca tienen todo bajo control, el 28,52\% (79) algunas veces, el $20,58 \%$ (57) sienten que nunca tienen todo bajo control.

Frente a la frecuencia con que han estado enfadados porque las cosas que le han ocurrido estaban fuera de su control, se encontró que el 32\% (89) algunas veces lo ha sentido. Se evidenció que el $65,83 \%$ (183) de los conductores expresaron que nunca y casi nunca han sentido que las dificultades se acumulan tanto que no pueden superarlas (Tabla 2).

Tabla 2. Distribución del estrés percibido según escala EEP-10 de los conductores de buses de una empresa de la ciudad de Medellín.

\begin{tabular}{lccc}
\hline \multicolumn{1}{c|}{ Variable } & $\begin{array}{c}\text { Frecuencia } \\
\text { absoluta }\end{array}$ & Porcentaje (\%) \\
\hline Ha estado afectado por algo que ha ocurrido inesperadamente & \\
Nunca & 119 & 42,8 \\
Casi nunca & 67 & 24,1 \\
Algunas veces & 84 & 30,2 \\
A menudo & 6 & 2,16 \\
Muy a menudo & 2 & 0,72
\end{tabular}

\section{Se ha sentido incapaz de controlar las cosas importantes en su vida}

Nunca

Casi nunca

Algunas veces

A menudo

Muy a menudo

Se ha sentido nervioso o estresado
130

64

56

21

7 
INVESTIGACIONES ANDINA No. 38, Vol. 21

$\begin{array}{lcc}\text { Casi nunca } & 57 & 20,5 \\ \text { Algunas veces } & 119 & 42,8 \\ \text { A menudo } & 27 & 9,71 \\ \text { Muy a menudo } & 17 & 6,12\end{array}$

Ha estado seguro sobre su capacidad para manejar sus problemas personales

Nunca

99

Casi nunca

Algunas veces

A Menudo

Muy a menudo

Ha sentido que las cosas le van bien

Nunca

Casi nunca

Algunas veces

A menudo

Muy a menudo

Ha sentido que podría afrontar todas las cosas que tenía que hacer

Nunca

Casi nunca

Algunas veces

A menudo

Muy a menudo

Ha podido controlar las dificultades de su vida

Nunca

Casi nunca

Algunas veces

A menudo

Muy a menudo

Ha sentido que tenía todo bajo control 


\begin{tabular}{lcc} 
Algunas veces & 79 & $28,52 \%$ \\
A Menudo & 12 & $4,33 \%$ \\
Muy a menudo & 10 & $3,61 \%$ \\
Ha estado enfadado porque las cosas que le han ocurrido estaban fuera de su control \\
Nunca & 65 & $23,38 \%$ \\
Casi nunca & 70 & $25,18 \%$ \\
Algunas veces & 89 & $32,01 \%$ \\
A menudo & 30 & $10,79 \%$ \\
Muy a menudo & 24 & $8,63 \%$ \\
Ha sentido que las dificultades se acumulan tanto que no puede superarlas & \\
Nunca & 104 & $37,41 \%$ \\
Casi nunca & 79 & $28,42 \%$ \\
Algunas veces & 63 & $22,66 \%$ \\
A menudo & 19 & $6,83 \%$ \\
Muy a menudo & 13 & $4,68 \%$ \\
\hline
\end{tabular}

\section{Análisis bivariado}

Se analizó la posible correlación de algunas características sociodemográficas y factores laborales con el estrés y se encontró una correlación positiva $y$ significativa $(0,232$ y valor $\mathrm{p}=0,000)$ entre la edad y el porcentaje de no control de estrés indicando que, a medida que aumenta la edad se controla menos el estrés.

Se encontró asociación entre la variable porcentaje de control de estrés con la ruta de buses que conduce el conductor (valor $\mathrm{p}=0,019$ ) según la prueba de Kruskal Wallis.

\section{Discusión}

El estrés laboral se define como la reacción que puede tener el individuo frente a las exigencias y presiones laborales que no se ajustan a sus conocimientos y capacidades, pocas oportunidades para tomar decisiones o ejercer control, poniendo a prueba su capacidad para afrontar la situación. Por esta razón, un trabajador que se encuentre estresado suele enfermarse con más frecuencia, tener poca o ninguna motivación, ser menos productivo y tener menor seguridad(18).

Al comparar el presente estudio con una 
trabajadores del volante de la ciudad de Chilpancingo, estado de Guerrero, México(19) se observan semejanzas en la edad promedio, la cual fue de 35,51 $(\mathrm{DE}=11,04$ años); similar al presente estudio donde el promedio fue de 36,53 años ( $\mathrm{DE}=11,86$ años). Con respecto al estado civil, se observaron diferencias: en el estudio realizado en México(19) el $10,99 \%$ eran solteros, el $68,06 \%$ casados y el $15,18 \%$ convivían en unión libre. Sucede de manera diferente al actual estudio donde el 28,4\% (79) eran solteros, el $29,1 \%$ (81) casados y el 38,1\% (106) convivían en unión libre.

En lo concerniente al tipo de violencia que sufren los conductores, el estudio actual revela una gran diferencia respecto al realizado en Madrid, España, sobre las condiciones de trabajo en el transporte público por carretera(20). En él, las formas más frecuentes de violencia que sufren los conductores son las amenazas que representaron un $8,2 \%$. Por el contrario, el presente estudió arrojó que, del total de los conductores que han sido víctimas de algún evento violento, el $56,4 \%$ (66) ha sido por atraco; el 15,3\% (186) por amenaza del tráfico vehicular y el $12,8 \%$ (20) ha sido víctima de dos eventos a la vez, como amenaza por vacunas, atraco y amenaza del tráfico vehicular.

Respecto al número de horas laboradas al día, se observa un comportamiento similar con otros estudios. En el estudio actual, el 88,09\% (244) trabajan más de 12 horas al día. Un estudio de Chile sobre riesgos psicosociales en conductores de transporte evidenció que las horas laboradas al día eran 11,4. Por otro lado, un estudio sobre riesgos cardiovasculares en conductores en la ciudad de Cali arroja un promedio de horas trabajadas diariamente de 13,4. También un estudio sobre ansiedad y estrés en conductores de transporte urbano de la cabecera departamental de Quetzaltenango reflejó que las jornadas laborales eran de 12 o más horas diarias $(21,10,22)$.

El estudio sobre el síndrome de burnout en conductores de transporte público de la ciudad de Arequipa(23) señala que el $62,8 \%$ lleva trabajando como conductor menos de cinco años, el 19,1\% lleva en este trabajo entre 5 y 10 años. Comparado con el presente estudio, se encontró un promedio de permanencia en la empresa de 9,5 años, con el tiempo mínimo de servicio en la empresa de 1 año y el máximo de 40 años.

Comparando el estudio actual y el estudio de Chile(21) en el que la suma de las categorías de sospecha y hallazgos de psicopatología fue de $26,9 \%$, se encontró en el presente estudio una proporción menor del 8,7\%, es decir, 24 conductores que alguna vez se le ha diagnosticado algún problema de salud mental.

Con respecto a la escala de estrés percibido en este estudio, se halló que el promedio de la calificación para el no control del estrés fue del 69\%, lo que indica que más de la mitad de las personas tienen dificultades para controlar el estrés. Estudios similares como el reportado en el artículo Estrés cotidiano en trabajadores del volante en México(19), encontraron un $26,7 \%$ de estrés en los conductores. En el estudio de Quetzaltenango(22) se evidenció que el 54\% de 
la población manifestaba un nivel bajo de estrés. Por otro lado, en el estudio de Arequipa(23) el 45,7\% presenta un nivel medio de síndrome de Burnout, mientras en el estudio sobre Burnout y carga mental en conductores realizado en Chile (24), solo el 23,22\% del total de la muestra percibieron niveles suficientemente elevados del Burnout.

El actual estudio permite evidenciar el poco tiempo disponible de los conductores para sí mismos y su familia; además, la manera en la que estas condiciones pueden verse afectadas por otros factores de orden social, laboral, político y económico. Por este motivo, generan un ambiente estresante que se verá reflejado en la atención que brinde al usuario.

Se recomienda fortalecer en estudios de carácter multifactorial y abordarse otras categorías de estudio, como el papel de

\section{Referencias}

1. Lazarus R, Valdés M, Folkman S. Estrés y procesos cognitivos. México: Ediciones Martínez Roca; 1986.

2. Martínez G. Todas las profesiones son estresantes. Fecha de consulta: 20 de mayo, 2008. Disponible en: http:// www.quasarcomunicacion.com.ar/ceeta/0423profesiones.htm

3. Organización Mundial de la Salud [OMS]. La organización del trabajo y el estrés. Protección de la salud de los trabajadores, serie núm. 3. Ginebra: OMS. 2004.

4. Cohen S, Kessler R, Underwood L. Measuring stress: A guide for health and social scientists. New York: Oxford University Press; 1997. 256 p. la familia, la empresa, las relaciones con los compañeros de trabajo y los perfiles de comportamiento, para esclarecer las condiciones ocupacionales de los conductores. Se recomienda, además, que los empresarios vinculen esfuerzos en un trabajo conjunto con el objetivo de disminuir la problemática presentada.

Agradecimientos: a los conductores por su participación y apoyo en el estudio; a las estudiantes Diana Isabel Ramírez, Francy Tatiana Lopera y Stephanie Bernate, a la gerente en sistemas de información en salud, Mónica Eliana Aristizábal y a la empresa de transporte público urbano por la acogida y colaboración.

Conflictos de interés: los autores no presentan conflicto de interés en la publicación de este artículo.

5. Gil P. Situación actual y perspectiva de futuro en el estudio del estrés laboral. Información psicológica. 2010;100:68-83.

6. OMS. Sensibilizando sobre el estrés laboral en los países en desarrollo: un riesgo moderno en un ambiente tradicional de trabajo. Protección de la salud de los trabajadores, serie núm. 6 Ginebra: OMS. 2008.

7. Durán M. Bienestar psicológico: el estrés y la calidad de vida en el contexto laboral. Revista Nacional de Administración. 2010. 1(1):71-84.

8. Aguilar-Zinser J, Irigoyen-Camacho M, Ruiz-García V, et al. Prevalencia de sobrepeso y obesidad en operadores mexi- 
canos del transporte de pasajeros. Gac Méd Méx. 2007; 143(1):21-25.

9. Arbeláez L, Delgado C, Giraldo I. Características clínicas y patológicas de conductores de una empresa de transporte público 2003. Investigaciones Andina. 2004;(8):14-21.

10. Camargo F, Gómez O, López M. Riesgo cardiovascular en conductores de buses de transporte público urbano en Santiago de Cali, Colombia. Revista Colombiana de Salud Ocupacional. 2013;3(3):18-22.

11. Göbel M, Springer J, Scherff J. Stress and strain short haul bus drivers: psychophysiology as a design-oriented method for analysis. Ergonomics. 1998;41(5):563-80

12. Chaparro P, Guerrero J. Condiciones de trabajo y salud en conductores de una empresa de transporte público urbano. Rev salud pública (Bogota). 2001;3(2):171-87.

13. Gamero-Burón C. Evaluación del coste por pérdida de jornadas laborales asociado al estrés laboral: propuesta para España. Estudios de Economía Aplicada. 2010; 28(3):1-20

14. Cohen S, Karmarck T, Mermelstein R. A global measure of perceived stress. J Health Social Behav. 1983;24(4):385-96.

15. Cohen S, Williamson G. Perceived stress in a probability sample of the United States. En: S. Scapan y S. Oskamp (eds). The social psychology of health: Claremont Symposium on applied social psychology. Newbury park, CA, pp: 31-67.

16. Campo A, Bustos G, Romero A. Consistencia interna y dimensionalidad de la Escala de Estrés Percibido (EEP-10 36 y EEP-14) en una muestra de universitarias de Bogotá, Colombia. Aquichan. 2009;9(3):271-80
17. Ministerio de Salud. Resolución 8430. Por la cual se establecen las normas científicas, técnicas y administrativas para la investigación en salud. Bogotá: Ministerio de Salud; 1993.

18. Gutiérrez AM, Viloria-Doria J. Riesgos psicosociales y estrés en el ambiente laboral. Revista Científica Salud Uninorte [Internet]. 2014 Mayo [Citado 2018 feb 13]; 30(1):1-5. Disponible en: http://rcientificas.uninorte.edu.co/index.php/salud/ article/view/6411/4757

19. Ramírez M, Hernández R, Reducindo $M$, et al. Estrés cotidiano en trabajadores del volante. Summa psicológica UST [Internet]. 2013 [Citado 2018 feb 13];10(1):85-90. Disponible en: http://pepsic.bvsalud.org/scielo.php?script=sci_arttext\&pid=S0719-448x2013000100008\&I$\mathrm{ng}=\mathrm{pt} \&$ tlng=es

20. Ordaz E, Maqueda J. Condiciones de trabajo en el transporte público por carretera. Medicina y seguridad para el trabajo [Internet]. 2014 Mar [citado 2018 feb 20];60(234): 90-98. Disponible en: http://dx.doi.org/10.4321/S0465546X2014000100008

21. Silva H, Lefio Á, Marchetti Nella, Benoi P. Riesgos psicosociales en conductores de transporte de carga y pasajeros urbanos e interurbanos, y su asociación con la autopercepción de salud y siniestralidad laboral. Cienc Trab [Internet]. 2014 Ago [citado 2018 feb 20]; 16(50):67-74. Disponible en: http://dx.doi.org/10.4067/S071824492014000200002

22. Coyoy G. Ansiedad y estrés en conductores de transporte urbano de la cabecera departamental de Quetzaltenango. [Tesis de grado]. Quetzaltenango, Guatemala: Universidad Rafael Landívar; 2014. $95 \mathrm{p}$.

23. Mendoza del Solar, L., Arias Gallegos, W., Masías Salinas, M. Síndrome de burnout en conductores de transporte público de la ciudad de Arequipa. Revista Peruana De PsicologíA Y Trabajo Social, 2(2), 
111-122. [Internet]. 2013. Consultado de http://revistas.uigv.edu.pe/index.php/psicologia/article/view/10

24. Olivares V, Jélvez C, Mena L, Lavarello J. Estudios sobre burnout y carga mental en conductores del trans- porte público de chile (Transantiago). Cienc Trab. [Internet]. 2013 dic [citado 2018 feb 20];15(48):173-8. Disponible en: $\quad$ http://dx.doi.org/10.4067/S071824492013000300011 
